\title{
S wie Spezialisten oder wo bleiben die Generalisten
}

\section{Dominik Heim}

PD Dr. med., Klinik Hohmad, Thun, Mitglied FMH

Es ist wie im Episodenfilm Kiki - el amor se hace (Paco Leon, 2016): Erzählt werden einzelne Geschichten, die am Schluss ein gemeinsames, stimmiges Bild ergeben. Dort geht es um Liebe und Sex, hier geht es um die Medizin (heute):

Das Mail war unmissverständlich: Nach 4 Wochen war bei Beschwerdearmut und unveränderter Frakturstellung der Clavicula noch wenig von Kallus zu sehen. Einen Monat vorher hatte mich ein Kollege um meine Meinung gebeten, ob man hier konservativ vorgehen könne. Ich bejahte. Dann ging er (leider) in die Ferien. Sein Stellvertreter schickte jetzt die Kontrollbilder: Wenn ich nicht innert 5 Tagen antworten würde, würde man für das weitere Vorgehen einen orthopädischen Schulterspezialisten beiziehen. Ich bat um Geduld für die Fraktur. Heute erhielt ich dann den Bericht des in der Zwischenzeit doch konsultierten Schulterspezialisten. Die Fraktur tat in der Zwischenzeit (auch radiologisch) tatsächlich ihren Willen zur Heilung kund, und der Schulterspezialist pflichtete dem konservativen Vorgehen bei. Ja, ich war ja schon erleichtert! Da war dieser Patient mit den Hämorrhoiden. Wie üblich schickte ich ihn zu einer Koloskopie zum Ausschluss einer proximalen Pathologie und mit der Bitte zur Evaluation einer chirurgischen Therapie. Leider war mein medizinischer Kollege unterdessen pensioniert. Gestern erhielt ich den Bericht seines Nachfolgers. Der Patient hätte nach mehreren Gummibandligaturen noch einige Resthämorrhoiden, aber bei Problemen könne er sich ja wieder melden. Ich war erstaunt! Ob die Chirurgie nicht doch eine valable Option gewesen wäre?

Vor zwei Jahren versorgte ich eine Unterschenkelfraktur mit einer durchgeschobenen LCDCP (limited contact dynamic compression plate) an der Tibia. Acht Wochen später wurde der Patient elsewhere nochmals operiert. Die fehlende Fixation der Fibulafraktur im Übergang vom mittleren zum distalen Schaftdrittel wurde bemängelt. Die Fibula wurde (endlich) verplattet und an der Tibia eine "modernere» LCP (locking compression plate) eingebracht. Im Anschluss kam es zu einem Infekt. Man äusserte in der Folge den Verdacht auf einen Behandlungsfehler wegen der Fibula bei der Erstversorgung. Der Gutachter hat mich dann «freigesprochen».

Wir sind gemäss FMH-Ärztestatistik 2014 ein Land von Spezialisten, der Anteil der Generalisten in der Schweiz beträgt ganze 37,4\% [1]. Die Chirurgen gehören zu den Spezialisten. Es gibt aber auch chirurgische SpezialSpezialisten. Das Gleiche gilt für weitere operative Disziplinen wie zum Beispiel die Orthopädie. Und in gewissen Situationen braucht es diese auch! Aber nicht, weil «auch Ärzte essen müssen», wie kürzlich ein Kollege sarkastisch bemerkte. Der Ruf nach einem Spezialisten wird heutzutage effektiv immer lauter: Die Bevölkerung sagt - und vor allem glaubt - es immer mehr, und die Kassen sind - trotz oder wegen ihres Kostenmanagements - mit second opinions immer grosszügiger. Und so sehen sich die Generalisten immer mehr dem Vorwurf mangelnder Kompetenz ausgesetzt. Das steigert die Attraktivität dieser Berufsgattung, die in der ländlichen Gegend immer mehr verschwindet, nicht. Wie sagte doch Thomas Lilti (Le médecin de campagne): In Frankreich sitzen die Ärzte "surtout à Paris, et au bord de la mer» [2]. Bei uns fehlt einfach das Meer...

Generalisten auch in der Musik: Vor drei Tagen sprachen «Wale» und ich im ZigZag über die letzte, kurze CD von Leonard Cohen You want it darker. Wir waren uns einig, er, der Musikspezialist, und ich, der (musikalische) Generalist: Fantastisch! Wir diskutierten über Keith Jarrett, den Jazzpianisten, der uns mit Arbour Zena 1975 die einzigartige jazz-klassische Dichtung präsentiert hat. Und wir dachten an Keith Emerson, den Keyboarder und Pianisten, der im Frühling ganz leise gegangen ist, und uns 1971 mit der Rockgruppe Emerson, Lake and Palmer mit Pictures at an Exhibition (Mussorgsky) eine der aufregendsten Rockadaptionen der Klassik hinterlassen hat. Es werden - auch da-immer weniger! PS: In der CH-Band Chapter Eleven sitzt ein Neurologe am Keyboard.

\section{Literatur}

1 Hostettler S, Kraft E. Frauen- und Ausländeranteil nehmen kontinuierlich zu. Schweiz Ärztezeitung. 2015;96(13):462-69.

2 Heim D. Le médecin de campagne - der Film. Schweiz Ärztezeitung. 2016;97(36):1249-51. 